\title{
Recurrent Inhibition of Cat Phrenic Motoneurons ${ }^{1}$
}

\author{
J. LIPSKI, ${ }^{2}$ R. E. W. FYFFE, AND J. JODKOWSKI \\ Experimental Neurology Unit, John Curtin School of Medical Research, A. N. U. Canberra 2601, Australia, and Department of \\ Physiology, Medical Academy, Warsaw 00-927, Poland
}

\begin{abstract}
These experiments were performed to re-examine the presence of a recurrent inhibitory pathway to phrenic motoneurons in cats. Following intracellular staining of 49 phrenic motoneurons with horseradish peroxidase, initial axon collaterals were demonstrated in six axons (12\%). Intracellular recording and averaging of the synaptic responses evoked by stimulation of the phrenic roots with an intensity subthreshold for axons of the examined motoneurons revealed (in spinalized cats with dorsal roots cut) inhibitory postsynaptic potentials (i.p.s.p.s) in 11/28 (40\%) motoneurons (amplitude, $115 \pm 69$ (SD) $\mu \mathrm{V}$; latency, $3.0 \pm 0.6 \mathrm{msec}$; duration from 12 to $25 \mathrm{msec}$ ). In the vicinity of the phrenic nucleus, Renshaw-like units were extracellularly recorded, which fired high-frequency bursts of action potentials following the stimulation of the phrenic roots (burst duration, 8 to $21 \mathrm{msec}$ ). Antidromic activation of the $\mathrm{C}_{5}$ phrenic root reduced the firing probability of fibers in the $C_{B}$ root. The duration of the reduced firing probability corresponded to the duration of both the i.p.s.p.s and the Renshaw units' firing period. It is concluded that Renshaw inhibition is present in phrenic motoneurons. The inhibitory effects of this recurrent pathway may be greater than judged from the small amplitude of the i.p.s.p.s recorded in individual motoneurons, particularly when the whole phrenic pool is activated and when motoneurons are near threshold. It is suggested that one of the functions of the pathway is to control phrenic motoneurons supplying the dorsal part of the diaphragm through the $C_{6}$ root when the more ventral part of the diaphragm is strongly activated.
\end{abstract}

Anatomical and physiological data indicate that recurrent inhibition of spinal $\alpha$-motoneurons is mediated by initial motor axon collaterals synapsing on Renshaw inhibitory interneurons. However, different spinal motor nuclei in mammals exhibit large variations with regard to the presence of axon collaterals (see "Discussion" for references). Similarly, in different motoneurons, recurrent inhibitory postsynaptic potentials (i.p.s.p.s) vary in amplitude or may not be present (e.g., Hultborn ct al., 1971; Rapoport, 1979; Egger et al., 1980). The differences seem to be related to the function of various motoneuron pools, although the degree of collateralization does not always allow predictions about the extent of the synaptic linkage and potency of inhibitory control (Evinger et al., 1979; Baldissera et al., 1981).

Received September 4, 1984; Revised December 6, 1984; Accepted December 7, 1984.

\footnotetext{
${ }^{1}$ We wish to thank Drs. S. Redman and A. Berger for their critical remarks on an earlier version of the manuscript and Mrs. Maureen Wiechert for typing the manuscript. The initial part of this study was supported by Grant 10.4 .03 of the Polish Academy of Science.

${ }^{2}$ To whom correspondence should be addressed.
}

The presence of a recurrent inhibitory pathway in spinal motoneurons supplying muscles with a pronounced respiratory function has been a matter of controversy. In two early intracellular studies of phrenic and intercostal motoneurons (Gill and Kuno, 1963; Sears, 1964), no significant degree of recurrent inhibition was detected and, subsequently, these motoneurons have been cited as examples which lack this type of inhibition (e.g., Eklund et al., 1964; Sears, 1966; Ellaway, 1971; Hultborn et al., 1971; Rapoport, 1979). The existence of recurrent inhibition in intercostal motoneurons has recently been re-examined by Kirkwood et al. (1981), who concluded that the inhibition is present in these motoneurons and that the strength of the overall inhibitory effect was comparable to that seen in hindlimb motor nuclei innervating long muscles at the ankle or knee.

The results of other recent studies on phrenic motoneurons are equivocal. Using intracellular injection of horseradish peroxidase (HRP), Cameron et al. (1983) found no initial axon collaterals in 11 cat phrenic motoneurons examined, while Webber and Pleschka (1976) reported a motor axon collateral in one out of four motoneurons stained with Procion Yellow. In an ultrastructural analysis of retrogradely HRP labeled phrenic motoneurons in the rat, Goshgarian and Rafols (1984) demonstrated an axon collateral in one cell. Electrophysiological studies also resulted in different conclusions. Thus, Gill and Kuno (1963) and Cameron et al. (1982) observed no recurrent i.p.s.p.s in phrenic motoneurons. On the other hand, Hilaire et al. (1983) recorded recurrent hyperpolarizing potentials in a few phrenic motoneurons, as well as Renshaw-like discharges in the phrenic nucleus.

The present experiments were undertaken to re-investigate the problem of whether recurrent inhibition and initial axon collaterals are present in cat phrenic motoneurons. Some preliminary results have been presented elsewhere (Lipski and Fyffe, 1984; Lipski and Jodkowski, 1984).

\section{Materials and Methods}

Animal preparation. Experiments were performed on 17 adult cats $(1.5$ to $3.4 \mathrm{~kg}$ ). Seven cats were initially anaesthetized with sodium pentobarbital (Nembutal; $40 \mathrm{mg} / \mathrm{kg}$ i.p.) and subsequently spinalized at $\mathrm{C}_{1}$ segment and decerebrated. No supplementary doses of Nembutal were given, and recordings were started a minimum of $6 \mathrm{hr}$ following the initial injection. The other 10 cats had their spinal cords left intact; six of these were anaesthetized with Nembutal throughout the experiment (with maintenance doses of 6 to $12 \mathrm{mg}$ i.v. given as required), while the remaining four cats were anaesthetized with chloralose and urethane (i.v., $40 \mathrm{mg}$ and $250 \mathrm{mg} / \mathrm{kg}$, respectively). All animals were placed prone in a stereotaxic frame with pelvic pins, and a vertebral clamp at $\mathrm{Th}_{1}$. They were paralyzed with gallamine triethiodide (Flaxedil; 5 to $10 \mathrm{mg} / \mathrm{kg} / \mathrm{hr}$ i.v.), artifically ventilated (end-tidal $\mathrm{CO}_{2}$ maintained close to $4 \%$ ), and subjected to a bilateral pneumothorax.

in all cats, the left $C_{5}$ and $C_{6}$ roots of the phrenic nerve were dissected free, cut distally, and prepared for standard bipolar stimulation and/or monophasic recordings of the multi-unit inspiratory activity. A laminectomy was performed at $\mathrm{C}_{4}$ to $\mathrm{C}_{7}$ level. All exposed dorsal roots on the left side were cut and reflected away from the entry zone, either at the start of the experiment or following initial determination of firing probability (see "Results"). 
A

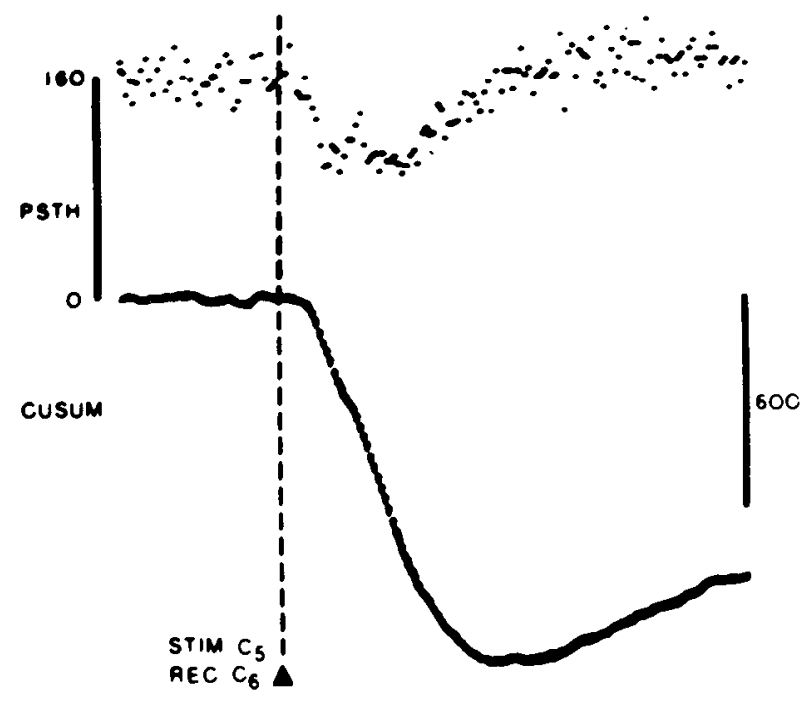

B

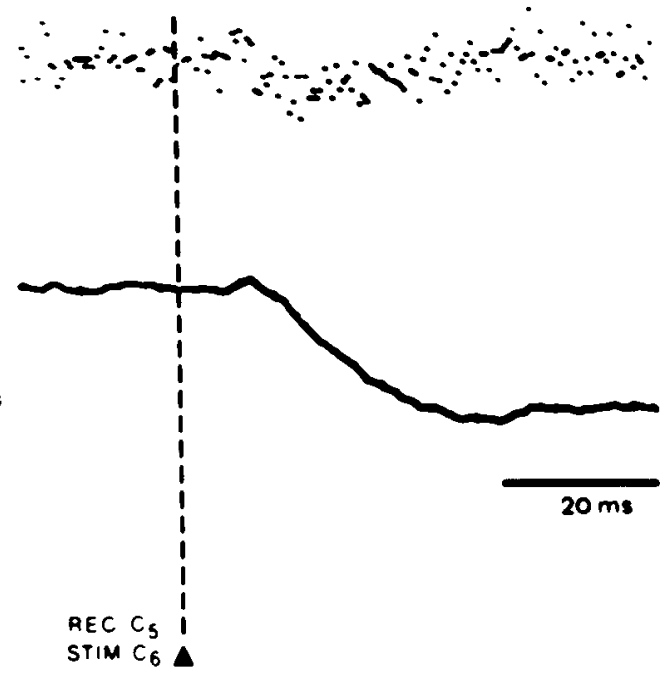

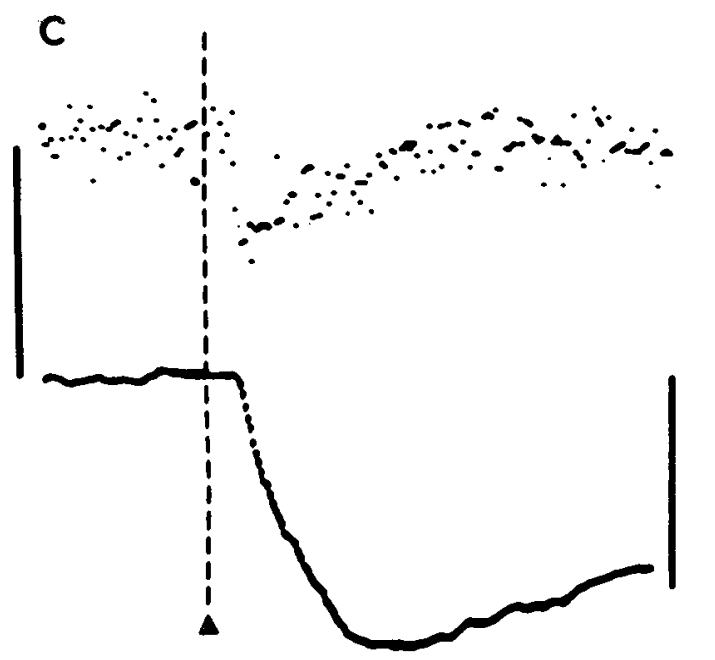

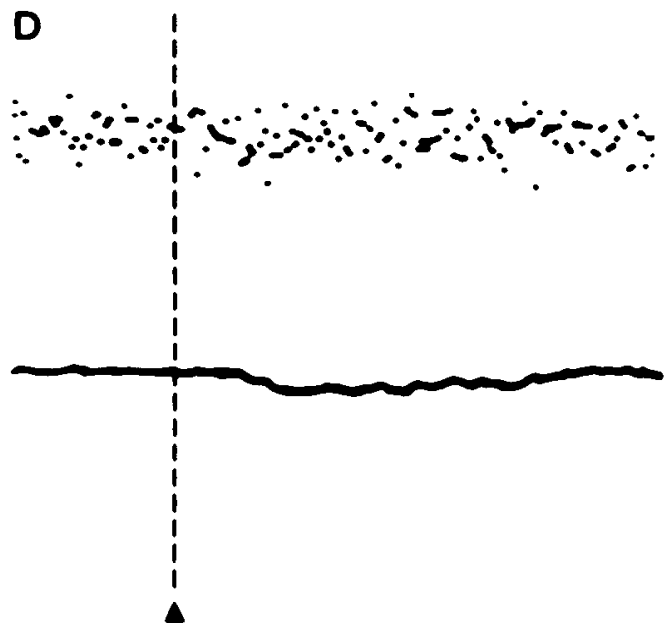

Figure 1. PSTHs and their CUSUMs of the activity recorded from the $C_{6}$ phrenic root following stimulation of the $C_{5}$ root $(A$ and $C)$ and from the $C_{5}$ root following the stimulation of the $C_{6}$ root $(B$ and $D)$. $A$ and $B$, Dorsal roots intact. $C$ and $D$, Dorsal roots $\left(C_{4}\right.$ to $\left.C_{7}\right)$ cut. Spinal cord intact. Bin width, 0.5 msec. Stimuli are marked by solid triangles and vertical dashed lines.

All exposed tissues were covered by warm mineral oil. Blood pressure in the femoral artery was continuously measured, and the animals' rectal temperature was held at 35 to $37^{\circ} \mathrm{C}$.

Stimulating and recording. In animals with their spinal cords intact, the multi-unit efferent inspiratory discharge was recorded monophasically from $\mathrm{C}_{5}$ or $\mathrm{C}_{6}$ phren nic roots. Threshold for spike discrirnination was set just above the noise level observed during expiration, and the firing probability of fibers in one phrenic root $\left(\mathrm{C}_{5}\right.$ or $\left.\mathrm{C}_{6}\right)$ was measured following stimulation $(0.05-\mathrm{msec}$ pulses) of the other root at $2 \mathrm{~Hz}$. The stimulus intensity was set to be three times threshold for the afferent volley recorded near the dorsal root entry zone of the same spinal segment. Both peristimulus time histograms (bin width, $0.5 \mathrm{msec}$ ) and their cumulative sums (Ellaway, 1978; Lipski et al., 1983) were recorded using 1000 to 3000 sweeps.

Intracellutar recordings were obtained using glass-capillary micro-electrodes with tips broken back to 1.0 to $2.0 \mu \mathrm{m}$ diameter and filled with either $2 \mathrm{M}$ potassium citrate and $150 \mathrm{~mm} \mathrm{KCl}$ ( 5 to 8 megohms) or, for intracellular staining, with a solution of $10 \% \mathrm{HRP}$ (Sigma type VI) in $0.1 \mathrm{M}$ Tris $\mathrm{HCl}$ buffer containing $0.5 \mathrm{M} \mathrm{KCl}$ at pH 8.6 (initial resistance, 10 to 20 megohms). These electrodes were also used for recording extracellular field potentials and unit discharges in the ventral horn of the $\mathrm{C}_{5}$ or $\mathrm{C}_{6}$ segment. The antidromic identification of phrenic motoneurons was described earlier (see Lipski et al., 1983). Each neuron was characterized by an antidromic latency and the threshold for excitation of its axon.

In motoneurons examined for recurrent i.p.s.p.s, the intracellular signal was recorded using $A C$ coupling (bandwidth, 1 or $10 \mathrm{~Hz}$ to $10 \mathrm{KHz}$ ), and the record was averaged over 500 to 1000 responses. In these experiments, the corresponding phrenic root was stimulated at an intensity just subthreshold for antidromic excitation of the impaled cell. Attempts to use stronger stimuli and to block antidromic spikes using short-lasting $(0.2$ to $1.0 \mathrm{msec})$ hyperpolaricing pulses (cf. Eccles et al., 1961) proved to be unsuccessful. This was due either to occasional discharge of an action potential at the break of the hyperpolarizing pulse or, more often, to a small residual depolarizing potential, presumably due to voltage-dependent changes in the membrane conductance. This potential had an amplitude (measured $5 \mathrm{msec}$ after the pulse) comparable with the size of the i.p.s.p.s (approximately 100 to $200 \mu \mathrm{V}$ ). No attempt was made in this study to measure the neuron input resistance or to change the amplitude and polarity of recorded intracellular potentials using polarizing current pulses.

HRP injection and histological techniques. HRP was ionophoretically injected using 8 to $20 \mathrm{nA}$ positive current pulses ( $400 \mathrm{msec}$ duration) at 2 $\mathrm{Hz}$. Injection attempts lasted from a few seconds to $30 \mathrm{~min}$. Generally, charge transfer had to be greater than $40 \mathrm{nA}$ times min for recovery of the injected neurons. Occasionally, in a single track or in closely spaced tracks ( $\leq 100 \mu \mathrm{m})$, more than one injection was attempted; the impaled cells were differentiated on the basis of antidromic latency and threshold measurements. This approach was adopted to label a large number of axons and to examine the anatomical relationships between neurons labeled close together. 


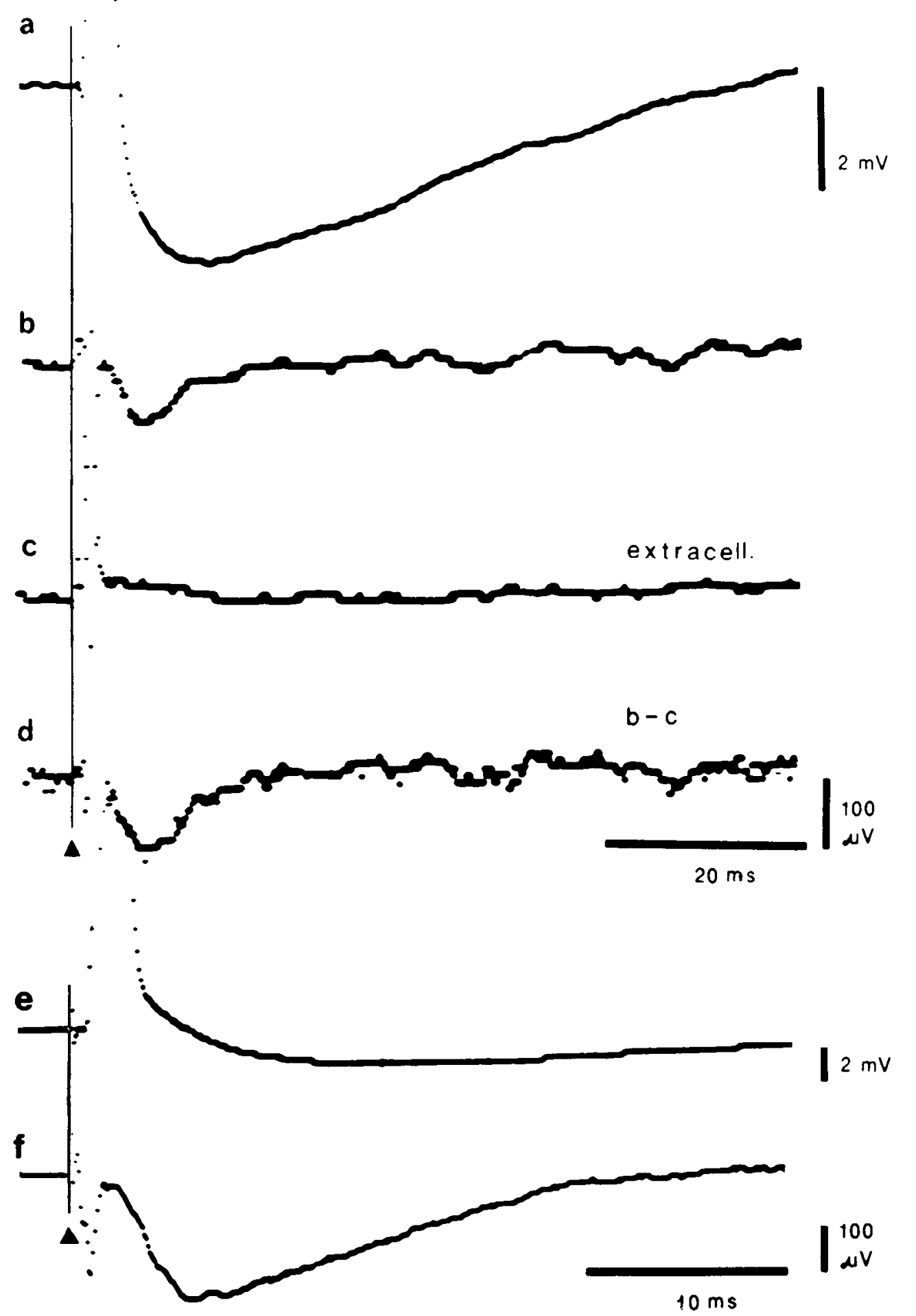

The histochemical methods have been described in detail elsewhere (Brown and Fyffe, 1984). Briefly, transverse sections were cut on a Vibratome at 50 to $80 \mu \mathrm{m}$, reacted with pyrocatechol and phenylenediamine (Hanker et al., 1977) for demonstration of the HRP reaction product, mounted serially on gelatin-coated slides, dehydrated, and then coverslipped for light microscopic analysis. Labeled phrenic motoneurons and their axons were examined under oil immersion at a total magnification of $\times 1000$ to detect the origin and trajectory of recurrent axon collaterals.

\section{Results}

Three types of experiments were performed in order to test for the presence of recurrent inhibition and to search for initial axon collaterals in phrenic motoneurons. In the first series, the firing
Figure 2. Potentials recorded from two phrenic motoneurons following stimulation of the corresponding phrenic root. Records a to $d$ were obtained from one neuron. Stimulus in a was just suprathreshold and in $b$ to $d$ just subthreshold for the axon of this motoneuron; $c$ is an extracellular record, and $d$ is the difference $(b-$ $c)$. Records $e$ and $f$ were obtained from another neuron; stimulus in e was just suprathreshold and in $f$ just subthreshold for its axon. Records $a$ and $e$ are the averages of 16 sweeps; records $b, c, d$, and $f$ are averages of 256 sweeps. Spinalized cats with dorsal $C_{4}$ to $C_{7}$ roots cut. Stimuli marked by filled triangles and vertical lines. The same amplitude calibration applies in averages $b, c$, and $d$. probability of one phrenic root was measured following antidromic activation of phrenic motoneurons in the adjacent segment. In the second series, intra- and extracellular recordings were made within the phrenic nucleus in an attempt to record recurrent i.p.s.p.s and Renshaw-like unit discharges. Finally, intracellular staining with HRP was performed in order to investigate the presence of axon collaterals.

Firing of phrenic motoneurons following antidromic activation of motoneurons in the adjacent segment. In four cats anaesthetized with chloralose-urethane and with the spinal cord intact, the efferent activity was recorded from one phrenic root $\left(\mathrm{C}_{5}\right.$ or $\left.\mathrm{C}_{6}\right)$ following the stimulation of the other root. Firing probabilities were measured 
$\mathbf{x}$

1.0

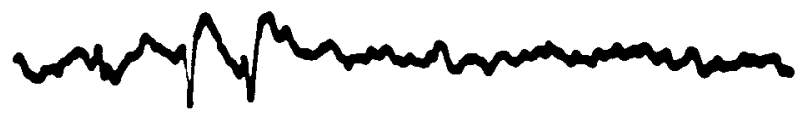

1.33

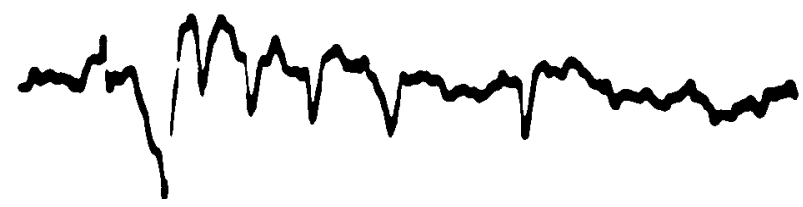

1.33

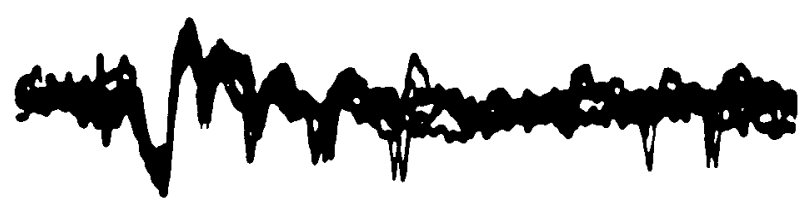

1.66

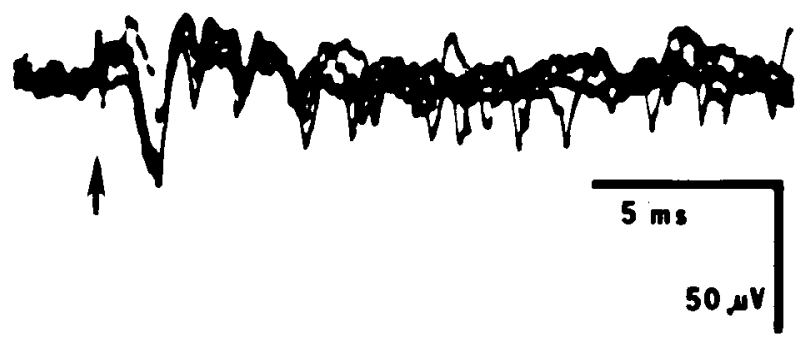

Figure 3. Renshaw cell-like discharge recorded in the $\mathrm{C}_{6}$ ventral horn following stimulation of the $C_{6}$ phrenic root. Spinalized cat with dorsal $C_{4}$ to $\mathrm{C}_{7}$ roots cut. Stimuli (arrow) at a strength given in multiples of threshold. Upper two records, single responses. Lower two records, six superimposed responses.

before and then after cutting the ipsilateral dorsal roots in four segments $\left(\mathrm{C}_{4}\right.$ to $\left.\mathrm{C}_{7}\right)$. An example of an altered discharge is showr in Figure 1. With the dorsal roots intact, stimulation of one phrenic root evoked only a weak short-latency autogenetic excitation of phrenic motoneurons in the adjacent segment. A more dominant effect was reduced firing (cf. Biscoe and Sampson, 1970), as shown in the peristimulus time histograms (PSTH). Cutting the dorsal roots almost completely eliminated the previously observed inhibition of the $\mathrm{C}_{5}$ root firing when the $\mathrm{C}_{6}$ root was stimulated (Fig. 1D). However, stimulation of the $C_{5}$ phrenic root still resulted in a pronounced inhibition of the activity in the $\mathrm{C}_{6}$ root (Fig. 1C). A similar asymmetry of the inhibitory responses of the $C_{5}$ and $C_{6}$ roots was observed in all of the animals when the dorsal roots were cut.

In order to describe the magnitude of the inhibition as a single parameter, the cumulative sums (CUSUM) of the bin counts in the PSTHs were measured (see, e.g., Ellaway, 1978; Kirkwood et al., 1981; Lipski et al., 1983). By adjusting the number of sweeps used to construct the PSTHs, the mean bin count in control bins (in the 20-msec period preceding the stimulus) was made the same ( $n=$ 160 ) in all of the original PSTHs. The minimum value of the CUSUM amplitude could then be used to express the strength of the inhibition (cf. Kirkwood et al., 1981). Measured in this way, the inhibition observed in the $C_{6}$ root of the four cats (with dorsal roots cut) was 960 (range, 710 to 1036 ), while the effect observed in the $C_{5}$ roots was 93 (range, 50 to 156). Thus, the mean ratio of the inhibition in the $\mathrm{C}_{5}$ root to that in the $\mathrm{C}_{6}$ root was 0.097 .

The mean latency measured from the stimulus to the beginning of inhibition in the $C_{6}$ root was 4.0 msec (range, 3.5 to 4.5 ). The mean duration of the inhibition, measured as the time from the onset of the CUSUM to its minimum, was $21.5 \mathrm{msec}$ (range, 17 to 27).

Intracellular and extracellular responses in the phrenic nucleus In seven cats, intracellular recordings were made from phrenic motoneurons in an attempt to record recurrent i.p.s.p.s. To eliminate descending supraspinal and segmental inputs and hence reduce the amount of synaptic noise, $\mathrm{C}_{4}$ to $\mathrm{C}_{7}$ ipsilateral dorsal roots were cut, and the cord was transected at $\mathrm{C}_{1}$.

Intracellular recordings with no obvious signs of neuronal injury (membrane potential more negative than $50 \mathrm{mV}$ and no spontaneous firing of action potentials) were made from a total of 28 phrenic motoneurons (12 in $C_{5}, 16$ in $C_{6}$ segments) for a period long enough to perform at least two averages of membrane potential using a minimum of 250 sweeps. Extracellular control recordings were made for the majority of tested motoneurons

Using a stimulus just subthreshold for the axon of each tested motoneuron examined, signal averaging revealed hyperpolarizing potentials in 11 out of 28 motoneurons (about $40 \%$ ). Three of these cells were recorded in the $C_{5}$ and eight in the $C_{6}$ segment. These hyperpolarizing potentials had amplitudes ranging from 45 to 260 $\mu \bigvee$ (mean \pm SD: $115 \pm 69$ ) and latencies of $3.0 \pm 0.6 \mathrm{msec}$. Their duration ranged from 12 to $25 \mathrm{msec}$.

An example is shown in Figure 2 (recorded in a $\mathrm{C}_{5}$ phrenic motoneuron) which had a peak amplitude of $78 \mu \mathrm{V}$ and a duration of approximately 14 msec. The true transmiembrane potential (the difference between the intracellular and extracellular traces; Fig. 2d) was $100 \mu \mathrm{V}$. Figure $2 f$ shows the largest potential recorded in this study.

The hyperpolarizing shifts of the membrane potential were presumed to be recurrent i.p.s.p.s. They were always substantially smaller than the hyperpolarizing potentials recorded when the corresponding phrenic root was stimulated with an intensity just suprathreshold for the axon of the impaled motoneuron (cf. Fig. 2, a and e). These latter hyperpolarizing potentials were the sum of small recurrent i.p.s.p.s and large after-hyperpolarizations. For the 11 phrenic motoneurons exhibiting recurrent inhibition, the mean ratio of the peak amplitude of the recurrent i.p.s.p.s to the peak amplitude of the hyperpolarizing potentials recorded in these motoneurons following suprathreshold stimulation of their axons was 0.04 .

Units which fired high-frequency bursts of action potentials following stimulation of the phrenic roots were occasionally detected during extracellular recording. Four of these Renshaw cell-like discharges were identified in the immediate vicinity of phrenic motoneurons, as judged by the presence of motoneuron antidromic field potentials. Two units were in the $\mathrm{C}_{6}$ segment and two in the $\mathrm{C}_{5}$. These recordings were unstable and of small amplitude (approximately $20 \mu \mathrm{V}$ ). A change in the position of the recording microelectrode of only 10 to $20 \mu \mathrm{m}$ resulted in the disappearance of the previously recorded unit. None of the four units was spontaneously active. The responses of one unit are shown in Figure 3. The discharge was evoked over the same stimulus range as the antidromic field potential. The unit responded with one or two spikes when the stimulus intensity was at threshold for the antidromic field. Increase in the stimulus intensity evoked longer bursts, and the maximum duration (16 msec) was observed at $1.66 \times T$. The maximum durations of the bursts in the other units were similar ( 8 to $21 \mathrm{msec}$ ). The mean response latency from the stimulus to the first spike evoked by supramaximal stimulation was $2.4 \mathrm{msec}$.

Figure 4. $A$ and $B$, The origin of phrenic motor axons from a proximal dendrite $(A)$ or soma $(B)$. The axon hillock (solid arrows) tapers to form a narrow initial segment (open arrows). $C$ to $E$. The initial parts of recurrent collaterals arising from three phrenic axons are shown, and their origins are indicated by curved arrows. In examples $C$ and $D$, the collateral branch originates at a node of Ranvier; in $D$, the internodal parts of the axon are faint and out of focus. Note the two adjacent cell bodies in $E$; the collateral arises from the initial segment of the axon originating from the upper of the two labeled neurons. Bar $=10 \mu \mathrm{m}$. 

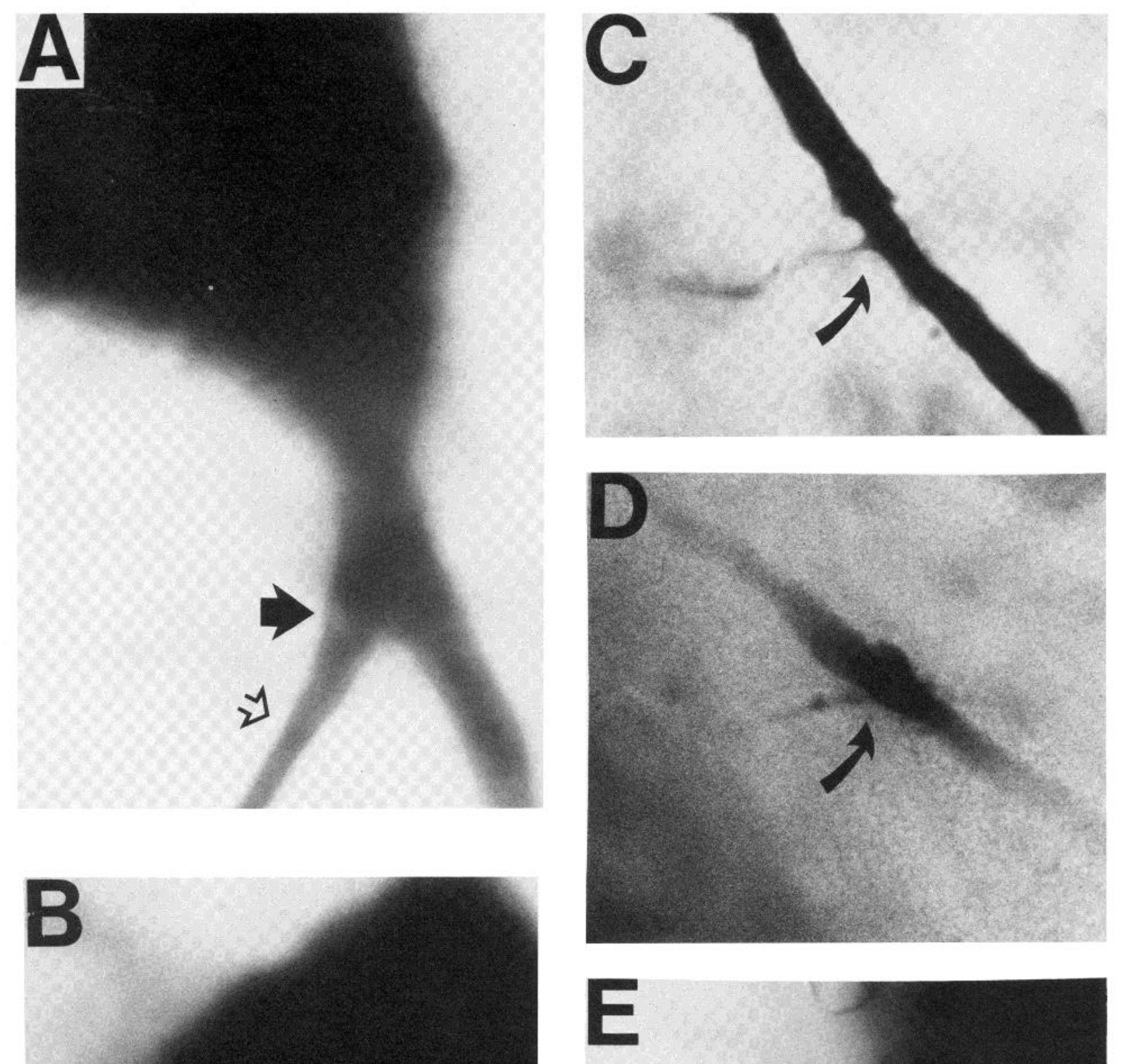

Figure 4 




Figure 5. Two collateral branches arising (at curved arrows) from a phrenic axon. The upper (more proximal) collateral courses dorsally and exhibits varicosities (arrowheads). The lower collateral is shorter and gives rise to three bouton-like enlargements (small arrows). The proximal and distal parts of the motor axon are out of focus in this montage and are indicated by open arrows. A dendrite from the neuron giving rise to these collaterals passes from left (lateral) to right (medial) between the two large arrows. Note that part of the dendrite which passes under the origin of the upper collateral is clearly at a different focal plane. Bar $=20 \mu \mathrm{m}$. 


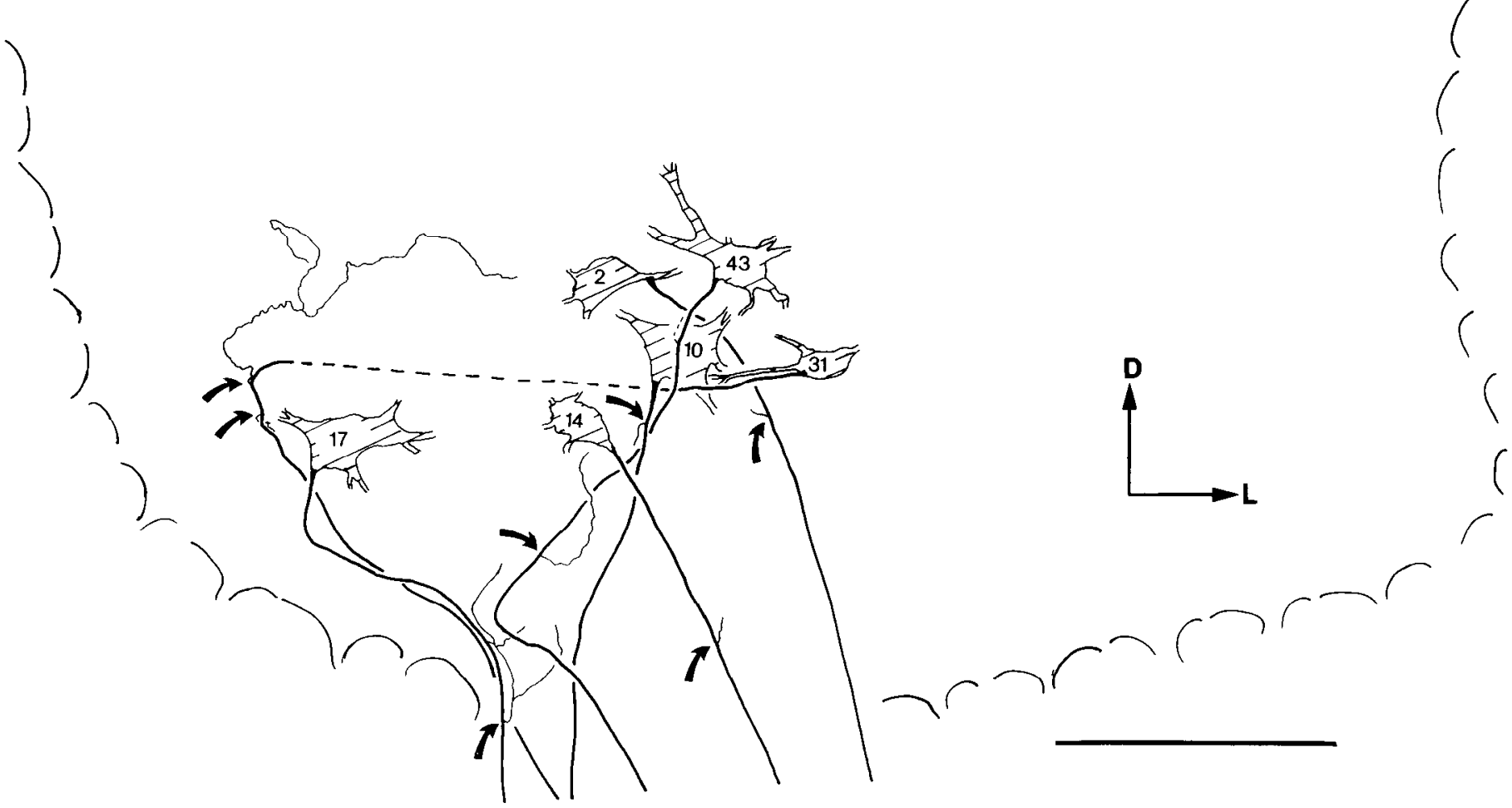

Figure 6. Reconstructions of the positions and axonal trajectories of the six phrenic motoneurons which issued axon collaterals. The cell numbers are referred to in the text. Axon collaterals are indicated by curved arrows. (The boundary of the ventral horn is an average representation of the six relevant locations). Note that cell 17 occupied an unusually medial position in the ventral horn. $D$, dorsal; $L$, lateral. $B a r=200 \mu \mathrm{m}$.

Intracellular HRP injections. A total of 70 intracellular injections (68 motoneurons in the $C_{5}$ segment and two in the $C_{6}$ segment) and two intra-axonal injections (phrenic motor axons in the $\mathrm{C}_{5}$ segment) were attempted. The majority of units were in $\mathrm{C}_{5}$, because our attention was concentrated on that segment in view of the high density of phrenic motoneurons at that level (Webber et al., 1979) and the electrophysiological evidence presented above, which indicated more pronounced recurrent inhibition from $C_{5}$ to $C_{6}$ motoneurons. Most of the injection sites were separated by about $500 \mu \mathrm{m}$ along the rostrocaudal axis of the cord, but in a few sites, staining of adjacent neurons was attempted with some success. Forty-nine neurons were recovered from the histological material ( $45 \mathrm{C}_{5}$ cells, two $\mathrm{C}_{6}$ cells, and two $\mathrm{C}_{5}$ axons). The intensity of staining was variable; 15 cells were judged to be well stained, 17 cells moderately stained, and 17 cells weakly stained or with evidence of extracellular deposit of HRP.

The axon of every stained cell could be identified and its trajectory could be traced in most cases from initial segment to exit in the ventral root. Seven axons (approximately 14\%) arose from a large proximal dendrite of the parent cell (e.g., Fig. 4A), while the remaining axons arose directly from the soma (e.g., Fig. 4B). The axonal trajectories shown in Figure 6 illustrate the range of courses taken: most run ventrally in direct fashion, while others travel medially at first and then loop ventrally. The mean diameter of axons in the grey matter was $4.7 \mu \mathrm{m}$ (range, 2.5 to $9.0 \mu \mathrm{m}$ ).

Six axons from $\mathrm{C}_{5}$ phrenic motoneurons had collateral branches (approximately $12 \%$ of the axons which were examined). One of these axons had two collaterals (Fig. 5 and Fig. 6, cell 31); one axon possibly had two collaterals (Fig. 6 , cell 43 ; the more proximal branch was difficult to define because of vascular damage), while the remaining four axons each issued one collateral (Fig. 6, cells 2, 10, 14 , and 17 and Fig. 4, C to E). All of the collaterals arose within the gray matter of the ventral horn or at the border between gray and white matter. They originated at distances of 30 to $450 \mu \mathrm{m}$ from the parent cell body, from nodes of Ranvier (Fig. 4, $C$ and $D$ ), or from the initial segment of the axon (Fig. 4E). Occasionally, the collaterals had a small cone-shaped hillock at their origin (e.g., Fig. 4C).

The collaterals were of fine diameter and in general could only be traced for relatively short distances (see Figs. 5 and 6). Indeed, only three collaterals (from two different axons) could be traced beyond their first branch point, and only one collateral (Fig. 5) had structures which we interpret as synaptic enlargements. Other collaterals (see also Fig. 5) had varicose swellings, at some distance from the termination of the branch, which were difficult to interpret. Because of the limited extent and staining intensily of the collaterals, it is not possible to comment definitively on the destination of these branches, but it seems that most of them ramify close to, or in, the phrenic motor nucleus, where they may terminate on Renshaw cells or phrenic motoneurons themselves (cf. Cullheim et al., 1977; Burke et al., 1982).

The position of the six labeled neurons which issued recurrent collaterals are plotted in Figure 6, and although these are selected cells, their locations are representative of the whole sample. It should be noted that one neuron (cell 17) is displaced medially from the main nucleus of cells. One other phrenic motoneuron in $\mathrm{C}_{5}$ had a similar location.

Our results on the pattern and extent of phrenic motoneuron dendrites are essentially in close agreement with the data of Cameron et al. (1983) in demonstrating rostrocaudal overlap between dendritic trees, dendrites which crossed the midline, and formation of bundles of dorsomedially and dorsolaterally oriented dendrites. However, some information was obtained as a consequence of labeling two or more identified motoneurons in close proximity. As has been noted previously with respect to the longitudinally oriented dendrites (Cameron et al., 1983), the dorsomedially and dorsolaterally oriented dendrites of adjacent motoneurons also formed bundles or fascicles and, within such fascicles, the dendrites of two different cells came into close apposition and remained so associated for tens or hundreds of microns (see Fig. 7). The identity of the dendrites comprising a fascicle was easy to determine in most cases, especially where the neurons were labeled to a different 


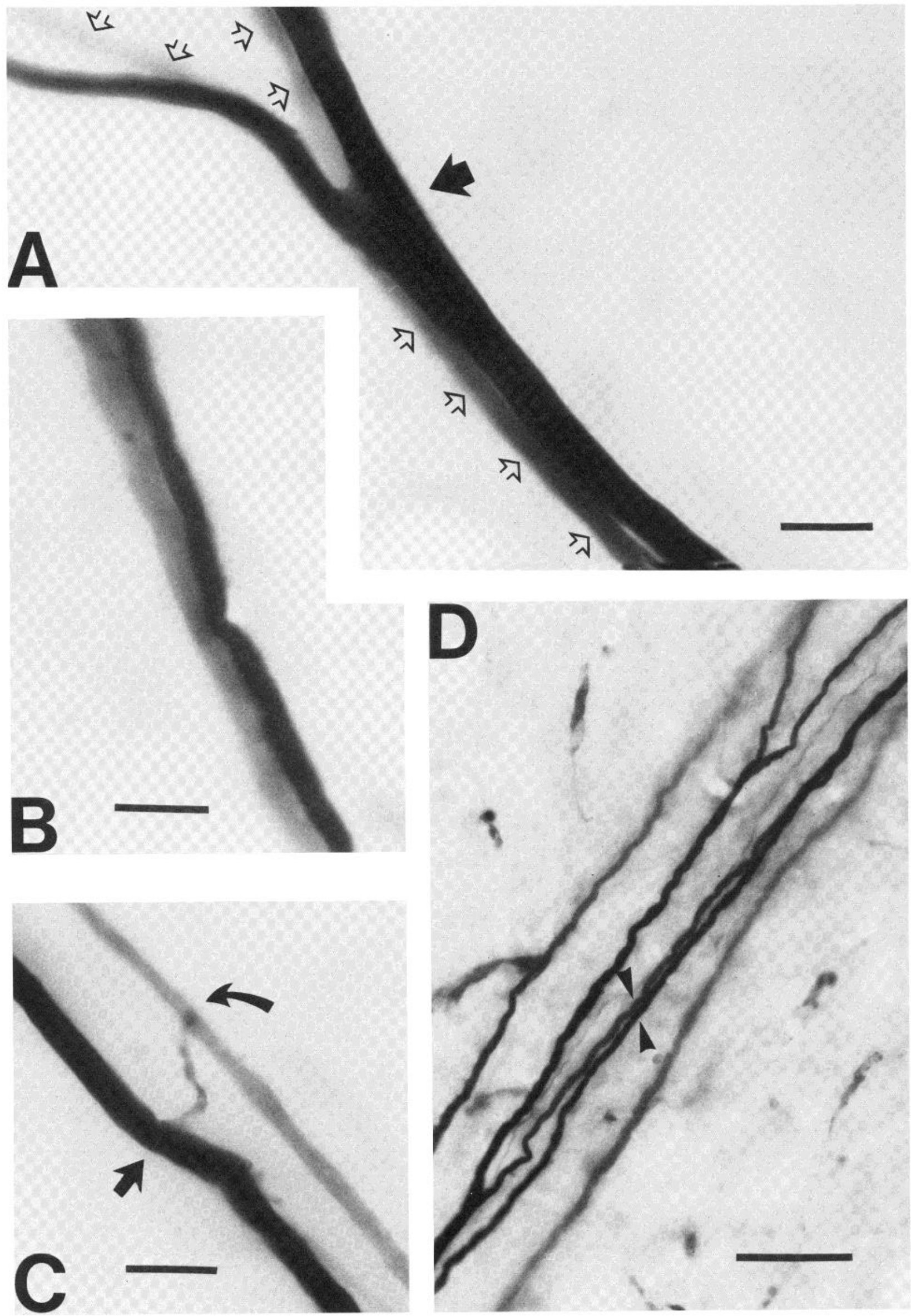


intensity, as exemplified in Figure 7. Close apposition (with no intervening space discernible using a $\times 100$ oil immersion objective) sometimes even continued beyond branch points of the respective dendrites. In Figure $7 A$, the two dendrites branch at the same locus, and the daughter branches continue to be apposed for some distance. In other interactions observed between dendrites from different neurons, there were dendritic appendages apparently bridging the gap between dendritic profiles (e.g., Fig. 7C).

\section{Discussion}

These experiments provide both morphological and electrophysiological evidence for a recurrent inhibitory pathway to phrenic motoneurons in cats. Recurrent inhibitory effects were observed in a relatively large proportion of these motoneurons, in spite of a low number of phrenic motor axons giving rise to initial axon collaterals. The morphological findings will be discussed tirst.

Our anatomical data demonstrate that approximately $12 \%$ of $\mathrm{C}_{5}$ phrenic motoneurons have axon collaterals. The absolute proportion may be underestimated due to variable, sometimes faint, staining of some phrenic motoneuron axons. On the other hand, although all of the collaterals observed arose from the moderately stained or wellstained axons, there were certainly a number of very well-stained axons which did not have recurrent collaterals. Additionally, only a few $C_{6}$ phrenic motoneurons are included in the present material, and it remains to be determined whether (as will be discussed below for the electrophysiological evidence) there is an asymmetry between $\mathrm{C}_{5}$ and $\mathrm{C}_{6}$ segments in the occurrence of axon collaterals.

The presence of phrenic motoneuron axon collaterals was suygested previously by Webber and Pleschka (1976), who, however, only presented an unconvincing illustration of a collateral arising from a Procion Yellow-stained neuron (see their Figure $4 A$ ). There is also ultrastructural evidence of an axon collateral in the phrenic nucleus of the rat (Goshgarian and Rafols, 1984). However, in the most detailed study to date of phrenic motoneuron morphology, no recurrent collaterals were detected (Cameron et al., 1983). The differences between the latter data and our results may be due to the respective sample sizes; 11 axons were examined by Cameron et al. (1983), whereas 49 axons were traced in the present study.

The frequency of occurrence of phrenic motoneuron recurrent collaterals in $\mathrm{C}_{5}$ segment may be compared with that in other motoneuron pools. In some motoneuron species, initial axon collaterals are given off by all or almost all axons (e.g., in triceps surae, tibialis anterior, quadriceps, posterior biceps, and dorsal neck and shoulder muscles; Burke et al., 1982; Cullheim and Kellerth, 1978a,b; P. K. Ruse and S. A. Keirstead, personal communication), while in others, axon collaterals are few in number (e.g., in sacral motoneurons mediating cutaneous spinal reflexes; Egger et al., 1980), or completely absent (e.g., in motoneurons of short plantar muscles of the foot; Cullheim and Kellerth, 1978a). Segmental location may partly determine collateral frequency, because it has been observed by $P$. K. Rose (personal communication) that, whereas trapezius motoneurons in $\mathrm{C}_{2}$ and $\mathrm{C}_{3}$ segments almost all have axon collaterals, trapezius motoneurons located more caudally in $\mathrm{C}_{5}$ and $\mathrm{C}_{6}$ segments (i.e., the same level as the phrenic nucleus) have a low frequency of occurrence of collaterals.

Although the main aim of the present experiments was to determine the presence or absence of axon collaterals, we have included in this paper some novel data which supplement the previous descriptions of phrenic motoneuron dendritic anatomy (see Cameron et al., 1983). In particular, extensive close appositions and crossbridges were apparent at the light microscopic level between the dorsomedially and dorsolaterally oriented bundles of dendrites arising from adjacent phrenic motoneurons. The role of the crossbridges and appositions require further study, since Lipski (1984) has shown that there is no detectable electrotonic coupling between phrenic motoneurons. Bundling of dendrites from different motoneurons might be a means by which synchronous activation of the motoneurons, mostly by synaptic inputs from brainstem premotor neurons, is attained.

The relatively low percentage of phrenic motoneurons with axon collaterals corresponded well to the low number of Renshaw-like units found in this study and in the recent report by Hilaire et al. (1983). These Renshaw units fired bursts of spikes at stimulus strengths appropriate to excite $\alpha$-motor axons. The burst duration corresponded to the duration of both the hyperpolarizing potentials recorded in individual phrenic motoneurons and the reduced firing probability of the whole phrenic root. Since the dorsal roots were cut, the effects could not be evoked through the primary afferents. Ventral root afferents are mainly unmyelinated (Coggeshall, 1980) and, since relatively low stimulus intensities were used, are extremely unlikely to be responsible for the observed responses. Therefore, we interpret our results as evidence for a recurrent inhibitory pathway involving phrenic motor axon collaterals, Renshaw units, and the target phrenic motoneurons.

Recurrent i.p.s.p.s were detected in approximately $40 \%$ of phrenic motoneurons. This number gives only a rough estimate of the proportion of phrenic motoneurons receiving recurrent inhibitory control, and it is probably underestimated due to the conditions of antidromic stimulation, in which the stimuli were subthreshold for the axons of the tested motoneurons. Assuming that the intracellular recordings were biased toward larger motoneurons, one can expect that stimuli subthreshold to their axons were also subliminal to the majority of axons in the same phrenic root. This would therefore decrease the amount of activation of the recurrent inhibitory pathway and could reduce both the number of inhibited motoneurons and the amplitude of the i.p.s.p.s recorded in individual motoneurons. The mean amplitude of the recurrent i.p.s.p.s recorded in this study $(115 \mu \mathrm{V})$ was still clearly greater than the average "unitary" effect of Renshaw cells on lumbar and sacral motoneurons (being $12.7 \mu \mathrm{V}$ ) reported by van Keulen (1981). With the assumption that inhibitory effects of single Renshaw cells on phrenic motoneurons are of similar magnitude, the inhibitory potentials recorded in our study must have been the result of activation of more than one inhibitory connection.

The size of the i.p.s.p.s recorded in this study was also affected by the base line membrane potential. Spinalization removed the rythmical depolarizing effects of descending activity. This decreased the driving force for the current at the recurrent inhibitory synapses, which normally would be active in inspiration (i.e., when the phrenic motoneurons are depolarized in intact spinal cords). On the other hand, spinalization substantially reduced the overall synaptic noise in phrenic motoneurons and hence caused the recording conditions to be more favorable for detecting i.p.s.p.s of a small amplitude. In

Figure 7. Phrenic motoneuron dendrites. The micrographs show details of the assuciations formed belween the dorsomedially or dorsolaterally oriented dendrites of adjacent neurons. A, Close apposition between a lightly stained dendrite (open arrows) and a densely stained dendrite from another neuron. The solid arrow indicates the point at which the associated dendrites branch concurrently. One pair of branches continue in association, while the other pair (top left) diverge $20 \mu \mathrm{m}$ or so distal to the branch point. $B$. The distal continuation about $100 \mu \mathrm{m}$ from the branch point of one pair of daughter branches from the dendrites shown in $A$. In $C_{1}$ a dense dendrite gives rise to an appendage (solid arrow) which forms a cross-bridge to a lightly stained dendrite from another neuron. The presumed site of contact is indicated by the curved arrow. $D$. Dendrites from two labeled phrenic motoneurons forming a dendritic bundle projecting dorsolaterally through the ventral horn. Distal (toward top right) to the arrows, two dendrites from different motoneurons apparently make a series of appositions. Bar in $A$ to $C, 10 \mu \mathrm{m}$; in $D, 50 \mu \mathrm{m}$. 
an early electrophysiological study, Gill and Kuno (1963) could find no evidence that phrenic motoneurons were subjected to recurrent inhibitory action. Their negative results can most likely be explained by a high level of noise in their recordings (of the order of several millivolts; see Fig. 3 in Gill and Kuno, 1963) and by the fact that no averaging technique was used. In the more recent investigations by Hilaire et al. (1983), in which averaging was applied, recurrent i.p.s.p.s were detected in $5 \%$ of their sample of phrenic motoneurons. The i.p.s.p.s reported by these authors had an amplitude comparable with the largest recurrent i.p.s.p.s recorded in the present sludy. Therefore, it can be suggested that the lower percentage of phrenic motoneurons receiving recurrent inhibition reported in the study by Hilaire et al. (1983), compared with the level observed $(40 \%)$ in this present study, could be due to difficulties in detecting potentials smaller than about $100 \mu \mathrm{V}$ in motoneurons with large synaptic noise levels. Another factor to be considered is the use of Nembutal by Hilaire et al. (1983). This anaesthetic was reported to have a depressant action on the recurrent inhibitory connections in the spinal cord (Eccles et al., 1956).

The functional significance of the recurrent inhibitory pathway in controlling the discharge of phrenic motoneurons still remains to be established. It is possible that the inhibitory effect of this pathway may be greater than judged from the small amplitude of i.p.s.p.s recorded in individual motoneurons, particularly when the whole phrenic motoneuronal pool is strongly activated and when motoneurons are near threshold. In addition, in animals with the spinal cord intact, the recurrent action can be facilitated by descending supraspinal action on Renshaw cells (see Hultborn et al., 1979; Hilaire et al., 1983).

The recurrent inhibitory action was not restricted to one segment. In animals with the spinal cord intact, the inhibition of the spontaneous firing could be observed in one phrenic root following supramaximal stimulation of another root. This inhibitory effect was, however, much stronger in the $\mathrm{C}_{6}$ root than in the $\mathrm{C}_{5}$. The simplest explanation of this phenomenon would be a larger number of motor axons in the $\mathrm{C}_{5}$ root. This, however, has not been demonstrated in previous studies, and in fact, there is an indication that the superior phrenic branch contains a smaller number of fibers than the inferior branch (Duron, 1981). Other studies (Webber et al., 1979; RikardBell and Bystrzycka, 1980) described only relatively small differences in the number of phrenic motoneurons in $C_{5}$ and $C_{6}$ segments. Another factor to be considered is the possible difference in the size of phrenic motoneurons located in the two segments, since large motor axons tend to produce larger recurrent effects and small (or type S) motoneurons tend to exhibit larger recurrent i.p.s.p.s (Friedman et al., 1981). This, however, is also unlikely to be the main reason for the prominent asymmetry reported here, as it has been shown that phrenic motoneurons of small, medium, and large diameter are arranged at random within the nucleus (Webber et al., 1979; cf. also Rikard-Bell and Bystrzycka, 1980)

The $C_{5}$ phrenic motoneurons supply the pars sternalis and pars costalis of the diaphragmatic dome, whereas the motoneurons of the $\mathrm{C}_{6}$ segment innervate preferentially the dorsal portion of crura and dome (Duron et al., 1979). Thus, it can be suggested that the asymmetry in the recurrent inhibitory action has a functional role in limiting the activity of phrenic motoneurons supplying the dorsal part of the diaphragm when the sternal and costal parts are strongly activated. Similar selective inhibition of the dorsal part of the diaphragm has been observed following the activation of oesophageal vagal afferents (Duron et al., 1975). Further studies are needed to examine the significance of the recurrent pathway to phrenic motoneurons at both segmental and intersegmental levels.

\section{References}

Baldissera, F., H. Hultborn, and M. Illert (1981) Integration in spinal neuronal systems. In: Handbook of Physiology, Nervous System II, Part 1, J. M.
Brookhart and V. B. Mountcastle, eds., pp. 509-595, American Physiological Society, Bethesda, MD.

Biscoe, T. J., and S. R. Sampson (1970) An analysis of the inhibition of phrenic motoneurones which occurs on stimulation of some cranial nerve afferents. J. Physiol. (Lond.) 209: 375-393.

Brown, A. G., and R. E. W. Fyffe (1984) Intracellular Staining of Mammalian Neurones, Academic Press, Inc., New York.

Burke, R. E., R. P. Dum, J. W. Fleshman, L. L. Glenn, A. Lev-Tov, M. J. O'Donovan, and M. J. Pinter (1982) An HRP study of the relation between cell size and motor unit type in cat ankle extensor motoneurons. J. Comp. Neurol. 209: 17-28.

Cameron, W. E., D. B. Averill, and A. J. Berger (1982) Detailed morphology of cat phrenic motoneurons. Soc. Neurosci. Abstr. 8: 874.

Cameron, W. E., D. B. Averill, and A. J. Berger (1983) Morphology of cat phrenic motoneurons as revealed by intracellular injection of horseradish peroxidase. J. Comp. Neurol. 219: 70-80.

Coggeshall, R. E. (1980) Law of separation of function of the spinal roots. Physiol. Rev. 60: 716-755.

Cullheim, S., and J. -O. Kellerth (1978a) A morphological study of the axons and recurrent axon collaterals of cat $\alpha$-motoneurones supplying different hind-limb muscles. J. Physiol. (Lond.) 281: 285-299.

Cullheim, S., and J. -O. Kellerth (1978b) A morphological study of the axons and recurrent axon collaterals of cat sciatic $\alpha$-motoneurons after intracellular staining with horseradish peroxidase. J. Comp. Neurol. 178: $537-$ 558.

Cullheim, S., J. -O. Kellerth, and S. Conradi (1977) Evidence for direct interconnections between cat $\alpha$-motoneurons via the recurrent axon collaterais. A morphological study using intracellular injection of horseradish peroxidase. Brain Res. 132: 1-10.

Duron, $B(1981)$ Intercostal and diaphragmatic muscle endings and afferents. In Regulation of Breathing. Part 1, T. F. Hornbein, ed., pp. 473-540, Marcel Dekker Inc., New York, Basel.

Duron, B., M. C. Jung-Cailiol, and D. Marlot (1975) Effets des distensions étagées de l'oesopage thoracique sur l'activité spontanée du diphragme chez le chat et le lapin. C. R. Acad. Sci. 281: 1863-1866.

Duron, B., D. Marlot, N. Larnicol, M. C. Jung-Caillol, and J. M. Macron (1979) Somatotopy in the phrenic motor nucleus of the cat as revealed by retrograde transport of horseradish peroxidase. Neurosci. Lett. 14: 159163.

Eccles, J. C., R. M. Eccles, and P. Fatt (1956) Pharmacological investigations on a central synapse operated by acetyicholine. J. Physiol. (Lond.) 131: 154-169.

Eccles, J. C., R. M. Eccles, A. Iggo, and M. Ito (1961) Distribution of recurrent inhibition among motoneurones. J. Physiol. (Lond.) 159: 479-499.

Egger, M. D., N. C. G. Freeman, and E. Proshansky (1980) Morphology of spinal motoneurons mediating a cutaneous spinal reflex in the cat. J. Physiol. (Lond.) 306: 349-363.

Eklund, G., C. von Euler, and S. Rutkowski (1964) Spontaneous and reflex activity of intercostal gamma motoneurons. J. Physiol. (Lond.) 171: 139163.

Ellaway, P. H. (1971) Recurrent inhibition of fusimotor neurones exhibiting background discharges in the decerebrate and the spinal cat. J. Physiol. (Lond.) 216: 419-439.

Ellaway, P. H. (1978) Cumulative sum technique and its application to the analysis of peristimulus time histograms. Electroencephalogr. Clin. Neurophysiol. 45: 302-304.

Evinger, C., R. Baker, and R. A. McCrea (1979) Axon collaterals of cat medial rectus motoneurons. Brai Res. 174: 153-160.

Friedman, W. A., G. W. Sypert, J. B. Munson, and T. W. Fleshman (1981) Recurrent inhibition in type-identified motoneurons. J. Neurophysiol. 46: $1349-1359$

Gill, P. K., and M. Kuno (1963) Properties of phrenic motoneurones. J. Physiol. (Lond.) 168: 258-273

Goshgarian, H. G., and J. A. Rafols (1984) The ultrastructure and synaptic architecture of phrenic motoneurons in the spinal cord of the adult rat. $\mathrm{J}$. Neurocytol. 13: 85-109.

Hanker, J. S., P. E. Yates, C. B. Metz, and A. Rustioni (1977) A new, specific, sensitive and non-carcinogenic reagent for the demonstration of horseradish peroxidase. Histochem. J. 9: 789-792.

Hilaire, G., M. Khatib, and R. Monteau (1983) Spontaneous respiratory activity of phrenic and intercostal Renshaw cells. Neurosci. Lett. 43: 97-101.

Hultborn, M., E. Jankowska, and S. Lindström (1971) Contribution from 
different nerves to recurrent depression of la I.P.S.P.s in motoneurones. I. Physiol. (Lond.) 215: 637-664.

Hultborn, H., S. Lindström, and H. Wigström (1979) On the function of recurrent inhibition in the spinal cord. Exp. Brain Res. 37; 309403.

Kirkwood, P. A. T. A. Sears, and R. H. Westgaard (1981) Recurrent inhibition of intercostal motoneurones in the cat. J. Physiol. (Lond.) 319: 111-130.

Lipski, J. (1984) is there electrical coupling between phrenic motoneurons in cats? Neurosci. Lett. 46: 229-234.

Lipski, J., and R. Fyffe (1984) Evidence for recurrent axon collaterals in cat phrenic motoneurones. Proc. Aust. Physiol. Pharmacol. Soc. 15: 1631.

Lipski, J., and J. Jodkowski (1984) Evidence for recurrent inhibition of cat phrenic motoneurones. Neurosci. Lett. 15(Suppl. 15): S44.

Lipski, J., L. Kubin, and J. Jodkowski (1983) Synaptic action of R $\beta$ neurons on phrenic motoneurons studied with spike-triggered averaging. Brain Res. 288: 105-118.

Lipski, J., E. G. Merrill, and W. Janczewski (1983) Analog calculation of cumulative sums from peristimulus time histograms and application of the technique to the analysis of rhythmic discharge of lung afferents. J. Neurosci. Methods 7: 165-170.
Rapoport, S. (1979) Reflex connexions of motoneurons of muscles involved in the head movement in the cat. J. Physiol. (Lond.) 289: 311-327.

Rikard-Bell, G, and E. Bystrzycka (1980) Localization of phrenic motor nucleus in the cat and rabbit studied with horseradish peroxidase. Brain Res. 194: 479-483.

Sears, T. A. (1964) Some properties and reflex connections of respiratory motoneurones of the cat's thoracic spinal cord. J. Physiol. (Lond.) 175: 386-403.

Sears, T. A. (1966) The respiratory motoneurone: Integration at spinal segment level. In: Breathlessness, J. B. L. Howell and E. J. M. Campbell, eds., pp. 33-52, Blackwell Scientific Publications, Oxford.

Webber, C. L., Jr., and K. Pleschka (1976) Structural and functional characteristics of individual phrenic motoneurons. Pfügers Arch. 364: 113-121.

Webber, Ch.L., Jr., R. D. Wurster, and J. M. Chung (1979) Cat phrenic nucleus architecture as revealed by horseradish peroxidase mapping. Exp Brain Res. 35: 395-406.

Van Keulen, L. (1981) Autogenetic recurrent inhibition of individual spinal motoneurones in the cat. Neurosci. Lett. 21: 297-300. 